\title{
Protective Effects of Antioxidants on Kidney Disease
}

\author{
Ghahari $\mathrm{L}^{1}$ and Safari $\mathbf{M}^{2 *}$ \\ ${ }^{1}$ Aja University of Medical Sciences, Iran \\ ${ }^{2}$ Department of Anatomy, Research Center of Nervous System Stem Cells, Semnan University of Medical Sciences, Iran \\ *Corresponding author: Manouchehr Safari, Research Center of Nervous System Stem Cells, Department of Anatomy, Semnan, Iran
}

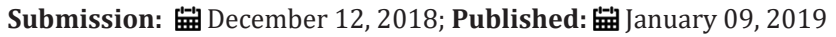

\section{Mini Review}

Oxidative stress is an imbalance between the amount produced and the removal of oxidants produced. In other words, the increase in reactive oxygen species (ROS) or reactive nitrogen species (RNS) and/or the reduction of the antioxidant/intrinsic/extrinsic is the main cause of this problem [1]. This is one of the most important causes of acute and chronic kidney disease [2]. Increasing the accumulation of free radicals within the kidney resulting from the malfunctioning of the antioxidant defense will ultimately lead to acute renal tubule necrosis, impaired function, and ultimately reduced glomerular filtration (GFR) [3]. The important factors of ROS molecules are hypochlorous acid, superoxide anion, hydrogen peroxide and hydroxyl radical. Because ROS molecules are very active, they quickly destroy molecules such as fats, DNA and proteins [4]. The factors that cause elevation of ROS and its products in the kidney cells are inflammatory cytokines, angiotensin II, mechanical pressure, hyperglycemia, protein kinase C (PKC), free fatty acids, TGF-beta1 and NADPH oxidases enzymes (NOXs). NADPH oxidases (NOXs) are enzymes that produce ROS. The NOX family contains seven subgroups (NOX1- 5 and DUOX 1 and 2) [5]. Of these seven types the NOX4 sever express in tubular cells, mesangial cells and podocytes. Increases of NOX4 expression has been shown in diabetic nephropathy, and on the contrary, shutting it off will protect the kidneys. Accumulation of $\mathrm{P}$-Cresyl sulfate in tubular cells causes over activation of PI3-K and PKC then trigger expression of Nox4and p22 phox and subsequently production of ROS. Enhancement of ROS production increase inflammatory cytokines and resulting tubular cell injury [6]. Oxidative stress induces apoptosis in renal tissue [7]. There are proteins that regulates Nox4 activity, such as Poldip2 (first known as NoxR1), and the p47phox-related adaptor protein Tks5 [8]. High glucose concentrations induce Nox4 protein. In cultured mesangial cells, high glucose in culture media stimulates a rapid expression of Nox4 protein, mitochondrial fraction, which is related to an increase of ROS inside the cell [9]. Although their performance on Nox4-mediated renal cell injury is unknown. Another important group that reduces or decreases the activity of antioxidants is RNS group. The main important RNS molecules are consisted of peroxynitrite, nitrite, and nitrate [10]. Antioxidants are divided into two important groups according to their mechanism of action, breaking and preventive antioxidants
[11]. Preventive antioxidants are able to dispose free oxygen and subsequently decrease the rate of chain initiation by break down of metals and finally subtractive hydroperoxides. Chain breaking antioxidants produce a stable product by donating or taking electrons from a free radical [11]. Medicinal herbs and some materials like Propolis are phytochemical compounds such as phenolic compounds or carotenoids that have potent antioxidant activity [12]. These natural materials protect cell membrane in the kidneys by reducing the lipid peroxidation (LPO) and increasing the endogenous antioxidants. Use of pargyline reduced apoptosis, necrosis, and fibrosis in kidney disease. This effect was related to reduction of the expression of collagen types I, III, IV, TGF- $\beta 1$, and induction of SOD1, catalase, and inflammatory factors [13]. The family of vitamin $\mathrm{E}$ is able to reduce the damage to proximal tubules by inhibiting the lipid peroxidation enzyme, increasing levels of GSH and activating the catalase. In addition, the NO2-/NO3 ratio improves. Etramethylpyrazine which found in grain and grain products is able to protect kidneys from ischemia/reperfusion injury. This protection is carried out by increasing the level of SOD enzyme, reduces malondialdehyde activity and then reduces the production of ROS [14]. Another group of compounds that is rich in tea, coffee, some fruits, cereals and vegetables, has a very powerful antioxidant activity by increasing the activity of $\mathrm{Cu} / \mathrm{Zn}$ SOD, glutathione peroxidase and catalase and to decrease the level of MDA [15]. Another antioxidant is $\alpha$-Lipoic acid. It is containing sulfur coenzyme which involved dehydrogenase reactions in mitochondria finally leading to (ATP) formation. In diabetic neuropathy, $\alpha$-lipoic acid through various mechanisms, they will protect the kidneys. Such as increase blood level and decrease ROS production in the distal nerves, reduces vascular endothelial growth factor (VEGF) protein in early diabetic nephropathy, and hypoxia in the retina. $\alpha$-lipoic acid prevent renal insufficiency, glomerular mesangial matrix expansion, and glomerulosclerosis by restoring glutathione and reducing MDA levels [16].

\section{Conclusion}

Oxidative stress is one of the most important factors in kidney damage. Generally, they act by increasing the production of oxidants or reducing the antioxidant defense of the cytosol, blood plasma, 
or through the failure to protect the cell membrane from lipid peroxidation. Antioxidants protect cells from damage by eliminating oxidative stress products as well as enhancing antioxidant defense.

\section{References}

1. Salmon AB, Richardson A, Pérez VI (2010) Update on the oxidative stress theory of aging: Does oxidative stress play a role in aging or healthy aging? Free Radic Biol Med 48(5): 642-655.

2. Sorg 0 (2004) Oxidative stress: A theoretical model or a biological reality? C R Biol 327(7): 649-662.

3. Rafieian Kopaei M, Baradaran A, Rafieian M (2013) Plants antioxidants: From laboratory to clinic. J Nephropathol 2(2): 152-153.

4. Valko M, Leibfritz D, Moncol J, Cronin MT, Mazur M, et al. (2007) Free radicals and antioxidants in normal physiological functions and human disease. Int J Biochem Cell Biol 39(1): 44-84.

5. Gorin Y (2013) Nox4 as a potential therapeutic target for treatment of uremic toxicity associated to chronic kidney disease. Kidney Int 83(4): 541-543.

6. Sedeek M, Nasrallah R, Touyz RM, Hébert RL (2013) NADPH oxidases, reactive oxygen species, and the kidney: Friend and foe. J Am Soc Nephrol 24(10): 1512-1518.

7. Wardle EN (2005) Cellular oxidative processes in relation to renal disease. Am J Nephrol 25(1): 13-22.

8. Diaz B, Shani G, Pass I, Anderson D, Quintavalle M, et al. (2009) Tks5-dependent, nox-mediated generation of reactive oxygen species is necessary for invadopodia formation. Sci Signal 2(88): ra53.
9. Meng D, Lv DD, Fang J (2008) Insulin-like growth factor-I induces reactive oxygen species production and cell migration through Nox4 and Rac1 in vascular smooth muscle cells. Cardiovasc Res 80(2): 299-308.

10. Ziech D, Franco R, Georgakilas AG, Georgakila S, Malamou-Mitsi V, et al. (2010) The role of reactive oxygen species and oxidative stress in environmental carcinogenesis and biomarker development. Chem Biol Interact 188(2): 334-339.

11. Benzie IF, Strain JJ (1999) Ferric reducing/antioxidant power assay: Direct measure of total antioxidant activity of biological fluids and modified version for simultaneous measurement of total antioxidant power and ascorbic acid concentration. Methods Enzymol 299: 15-27.

12. Sameni HR, Ramhormozi P, Bandegi AR, Taherian AA, Mirmohammadkhani M, et al. (2016) Effects of ethanol extract of propolis on histopathological changes and anti-oxidant defense of kidney in a rat model for type 1 diabetes mellitus. J Diabetes Investig 7(4): 506-513.

13. Rojas Rivera J, Ortiz A, Egido J (2012) Antioxidants in kidney diseases: The impact of bardoxolone methyl. Int J Nephrol 2012: 321714.

14. Khan MR, Siddiqui S, Parveen K, Javed S, Diwakar S, et al. (2010) Nephroprotective action of tocotrienol-rich fraction (TRF) from palm oil against potassium dichromate ( $\mathrm{K} 2 \mathrm{Cr} 2 \mathrm{O}$ 7)-induced acute renal injury in rats. Chem Biol Interact 186(2): 228-238.

15. Fan SH, Zhang ZF, Zheng YL, Lu J, Wu DM, et al. (2009) Troxerutin protects the mouse kidney from d-galactose-caused injury through anti-inflammation and anti-oxidation. Int Immunopharmacol 9(1): 91-96.

16. Obrosova IG, Fathallah L, Greene DA (2000) Early changes in lipid peroxidation and antioxidative defense in diabetic rat retina: Effect of DL-alpha-lipoic acid. Eur J Pharmacol 398(1): 139-146.
Creative Commons Attribution 4.0 International License

For possible submissions Click Here

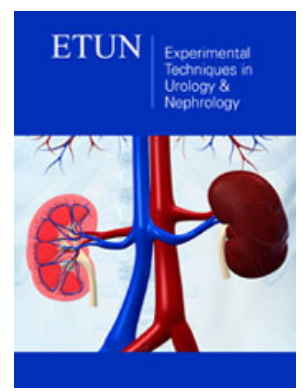

Experimental Techniques in Urology \& Nephrology

\section{Benefits of Publishing with us}

- High-level peer review and editorial services

- Freely accessible online immediately upon publication

- Authors retain the copyright to their work

- Licensing it under a Creative Commons license

- Visibility through different online platforms 